\title{
Early application of low-dose glucocorticoid improves acute respiratory distress syndrome: A meta-analysis of randomized controlled trials
}

\author{
ZHI-GANG YANG, XIAO-LI LEI and XIAO-LIANG LI \\ Department of Respiratory Medicine, Henan Provincial People's Hospital, Zhengzhou, Henan 450003, P.R. China
}

Received April 7, 2015; Accepted June 20, 2016

DOI: $10.3892 /$ etm.2017.4154

\begin{abstract}
Previous clinical trials have investigated the effect of glucocorticoid therapy in acute respiratory distress syndrome (ARDS), with controversial results, particularly with regard to the early administration of low dose glucocorticoid. The present meta-analysis aimed to assess whether the application of glucocorticoid was able to reduce mortality in patients with ARDS. A literature search was performed using online databases, including MEDLINE, Embase, Cochrane and CNKI regardless of whether the studies were published in English or Chinese. Following assessment via inclusion and exclusion criteria, two reviewers screened controlled randomized trials which investigated glucocorticoid therapy in ARDS patients and independently extracted data. The quality of all of the included trials was evaluated based on blinding, randomization and other methods. A total of 14 studies with 1,441 patients met the inclusion criteria. The results of the meta-analysis demonstrated that glucocorticoid significantly reduced the overall mortality of patients with ARDS [relative ratio (RR), 0.68 ; 95\% confidence interval (CI), 0.50-0.91; $\mathrm{P}<0.05]$, particularly with a low-dose of glucocorticoid (RR, 0.57; 95\% CI, $0.39-0.84 ; \mathrm{P}<0.05)$ at the early phase of ARDS (RR, 0.37; 95\% CI, 0.16-0.86; $\mathrm{P}<0.05)$, and a longer duration of steroids ( $R R$, 0.44; 95\% CI, 0.30-0.64; $\mathrm{P}<0.05)$. Administration of steroids also significantly increased the number of days that patients remained alive and were off mechanical ventilation (RR, 3.08; 95\% CI, 1.49-4.68; P<0.05) without significantly increasing the novel infection rate (RR, 1.00; 95\% CI, 0.44-2.25; $\mathrm{P}<0.05)$. Due to inconsistencies and other limitations, the quality of the studies used for the meta-analysis of the effect of glucocorticoid on mortality was low. In conclusion, early use of low dose glucocorticoid may effectively reduce mortality in patients with ARDS. However, this conclusion may be affected
\end{abstract}

Correspondence to: Dr Zhi-Gang Yang, Department of Respiratory Medicine, Henan Provincial People's Hospital, 7 Weiwu Road, Zhengzhou, Henan 450003, P.R. China

E-mail: yangzhigmedsci@163.com

Key words: glucocorticoid, acute respiratory distress syndrome, meta-analysis, effect, mortality by the limited quality of the studies included in the present meta-analysis.

\section{Introduction}

Acute respiratory distress syndrome (ARDS) is a life-threatening condition with non-cardiogenic permeability pulmonary edema characterized by the increased permeability of pulmonary capillary endothelial cells and alveolar epithelial cells, resulting in inflammation, hypoxemia and multiple organ failure $(1,2)$. Mortality rates from ARDS are estimated to be 34-64\%; however, with proper treatment, the mortality rate from ARDS may be reduced to $25 \%(2,3)$.

Numerous studies (4-7) have suggested that an excessive inflammation reaction may be responsible for this apparent mortality. The main causes of inflammation are inflammatory cytokines, including interleukin-1 (IL-1), IL-6, tumor necrosis factor- $\alpha$ (TNF- $\alpha$ ), C-reactive protein (CRP) and other factors (7). The release of these elevated cytokines contribute to the development of ARDS. Given that ARDS is associated with acute pulmonary inflammation, it is also expected to improve treatment outcomes of anti-inflammation therapies. However, the results of clinical studies using glucocorticoid or corticosteroid to cure ARDS remain controversial.

Although glucocorticoids have a powerful capability in suppressing the inflammatory process $(8,9)$, this effect is correlated with the magnitude and duration of inflammation $(7,10,11)$. Following the administration of glucocorticoid agents, patients who exhibited a significant reduction in TNF- $\alpha$ and IL-1 usually exhibited an improved outcome $(12,13)$; whereas persistent elevation of inflammatory cytokines predicted a poor outcome in patients with ARDS (11). These observations lead researchers to questions when and what doses of glucocorticoid should be used in the treatment of ARDS. Several reviews have investigated these problems $(7,12,14,15)$. One review demonstrated that corticosteroids did not significantly reduce hospital mortality when pooling across all trials (12); whereas another study reported that it was not clear whether a lower dose of glucocorticoid for persistent ARDS could reduce mortality in the long-term (16).

The present study aimed to determine the effects of different doses and application times of glucocorticoid on the mortality of patients with ARDS by conducting a meta-analysis of previous randomized control trials. 


\section{Materials and methods}

Literature search. All studies of interest were identified from electronic databases, including MEDLINE (https://www.nlm. nih.gov/bsd/pmresources.html), Embase (https://www.elsevier.com/solutions/embase-biomedical-research), Cochrane for English literature (http://www.cochranelibrary.com/), and CNKI (http://www.cnki.net/) and VIP database for Chinese literature up to November 2013. The following terms were used: Glucocorticoid, corticosteroid, methylprednisolone, hydrocortisone, acute respiratory distress, adult respiratory distress, ARDS, acute respiratory distress syndrome and acute lung injury. In addition to the randomized controlled trials, published reviews and relevant bibliographies from meetings such as the American Thoracic Society, Society of Critical Care Medicine, and the International Society of Intensive Care and Emergency Medicine were also searched and reviewed.

Inclusion and exclusion criteria. To avoid potential bias, the following inclusion criteria were used: i) Designed as randomized controlled trials; ii) participants aged $\geq 18$ years old with a clear diagnosis of ARDS regardless of etiology, race, nation, and sex; iii) trials should include an intervention arm receiving glucocorticoid treatment and a control arm receiving placebo or standard care. The dose and cycles of the glucocorticoid treatment and data of mortality were presented in published literature or available from authors. The exclusion criteria were as follows: (i) Animal, infant or children study subjects; ii) glucocorticoid therapy used to prevent the occurrence of ARDS but not to treat it; iii) definitive diagnosis standards of ARDS were not presented during the trials; and iv) detailed information about glucocorticoids, including the intervention period, dose and duration of administration were not provided in the literature.

Data extraction and quality assessment. Population data, number of participants, glucocorticoid administration route, mortality and other endpoints were independently extracted from the studies by two reviewers, and a third reviewer was introduced when disagreements occurred. Important missing data relevant to the study design was also sought, including the blinding method used. Subgroup analyses regarding the dose and duration of glucocorticoid in the treatment of ARDS was also conducted, since the dose and duration of glucocorticoid may be important factors that influence outcomes. The early period of ARDS was defined as the first seven days of the diagnosis of ARDS, and the late period as the seven days following diagnosis of ARDS, as described by Wajanaponsan et al (17). Low-dose or high-dose therapy was defined according to previous studies $(12,18,19)$.

Quality assessment of the evidence eligible for the present meta-analysis was performed by reviewers using a scoring method from a previous study (20). Differences in scoring were resolved by consensus and the extracted data were recorded carefully and independently verified by two investigators prior to the meta-analysis.

Data analysis. Relevant data extracted and confirmed by two authors were used for the meta-analysis. All data were typed using Review Manager 5.2 software provided by the Cochrane
Collaboration Group (Herlev, Denmark). For dichotomous data, the Mantel-Haenszel method was used to estimate the risk ratio (RR) with a 95\% confidence interval $(\mathrm{CI})$. The inverse variance method was applied to analyze continuous data and the mean differences had 95\% CIs.

Statistical analysis. In order to reduce the heterogeneity of the included studies, the heterogeneity of the clinic and methodology were assessed preferentially. In order to test statistical heterogeneity, the $\chi^{2}$ test was introduced. $\mathrm{P}<0.01$ was used to indicate a statistically significant difference. The $\mathrm{I}^{2}$ test was used to evaluate the pooled variation between all of the eligible trials. A random-effects model or a fixed-effects model was used to perform meta-analysis, and were selected according to the significance of the heterogeneity, as described previously (21). In addition, bias risk was also assessed based on the standards reported in the Cochrane Handbook (21). Statistical tests were performed using Stata software, version 12.0 (StataCorp LLC, College Station, TX, USA).

\section{Results}

Study flow. By limiting the search to human clinical studies and excluding animal studies and basic research, 227 articles were determined to be relevant to the topic of acute respiratory distress syndrome and glucocorticoids, and 190 citations were excluded after reviewing the titles and abstracts. The remaining 37 articles were reviewed in full and 14 articles (22-34) were included for further review. In these 14 studies, two trials $(31,33)$ did not provide mortality data in the text and, although the authors were contacted by e-mail, this data remained unavailable. A total of 14 articles were eligible for the final meta-analysis. A flowchart of the present meta-analysis is presented in Fig. 1.

Characteristics and quality of the studies included. According to the inclusion and exclusion criteria, there were 14 articles $(18,22-34)$, included in the final analysis, two of which were Chinese $(30,34)$. The basic characteristics and quality of the studies included are presented in Tables I and II. A total of 1,441 patients with ARDS were included, with 774 cases in the treatment group and the remaining 667 in the control. Standard care, mechanical ventilation, and other supportive care was applied to patients in both groups. Nine studies $(24-30,34)$ used low-dose therapy and high-dose therapy was studied in three trials $(22,23,32)$. There were 11 studies $(18,22-26,29,30,32-34)$ that defined as glucocorticoid intervention at the early phase of ARDS onset, and two studies $(27,28)$ were considered to treat ARDS with glucocorticoid during the late period. Furthermore, two studies $(27,28)$ provided data for the early and late treatment of ARDS. Illness severity scores were not applied in the present study.

As shown in Table II, the quality of the studies included was acceptable for the comparison of the effect of glucocorticoid therapy on the mortality of ARDS, although some studies $(18,25,29-31,34)$ failed to provide a clear method of blinding, and a few studies $(18,24,34)$ revealed limitations in the sample size, anti-inflammatory agents and in the risk of bias of glucocorticoid stopping for perceived improvement. 


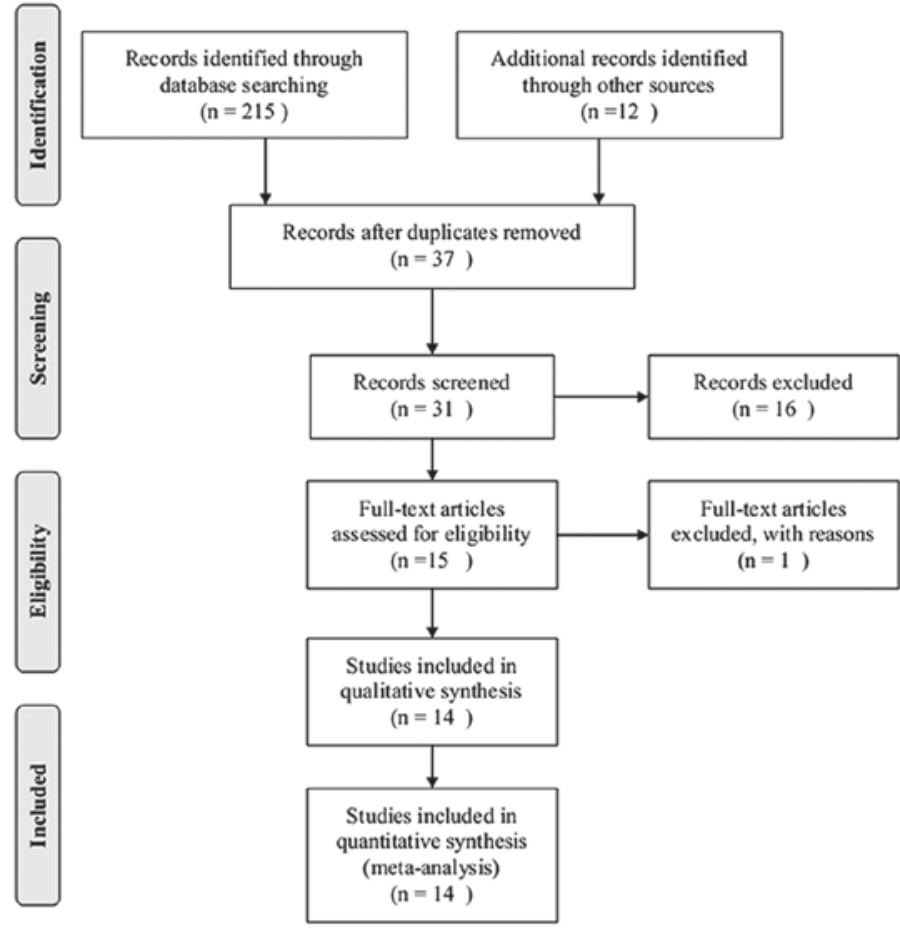

Figure 1. Flow diagram of studies.

Meta-analysis. Initially, a meta-analysis of the mortality, incident infection, days remained alive and off mechanical ventilation, lung injury scores, multiple organ failure and $\mathrm{PaO}_{2} / \mathrm{FiO}_{2}$ ratio was planned. However, due to the limited data available, only a pooled analysis of the mortality, incident infection and days alive and off mechanical ventilation was conducted. Subgroup analysis of the mortality data accordingly to the different doses and duration of glucocorticoid therapy was also included.

Effect of glucocorticoid on mortality in ARDS patients. A total of 12 trials $(18,22-30,32,34)$ were selected to assess whether glucocorticoid treatment was beneficial to patients with ARDS by reducing mortality. The main findings are depicted in Fig. 2, estimating a value of 0.68 for the RR of overall mortality $(95 \%$ CI, 0.50-0.91). Supported by the pooled analysis, a significant difference was identified in the glucocorticoid intervention group for lowering the overall mortality when compared with the control group $(\mathrm{P}<0.05)$.

As previously described, studies of low-dose and high-dose glucocorticoid treatment with a threshold value of $2 \mathrm{mg} / \mathrm{kg} / \mathrm{d}$ were identified. As presented in Fig. 2, there were 9 articles $(18,24-30,34)$ comparing the impact of low-dose treatment of glucocorticoid on mortality rates of ARDS patients with the controls. Significantly reduced mortality was identified in the low-dose intervention group compared with the control, and the combined RR was 0.57 with a $95 \%$ CI between 0.39 and $0.84(\mathrm{P}<0.05)$, indicating that treating ARDS with low-dose administration of glucocorticoid may sufficiently decrease the mortality of ARDS by a relative ratio of 0.57 . However, this result was no longer beneficial when comparing low-dose glucocorticoid with the control in late steroid rescue studies $(\mathrm{P}>0.05)$. Only three articles $(22,23,32)$ studied the effect of high-dose glucocorticoid on the mortality rates of ARDS, and the pooled data (Fig. 2) failed to support the hypothesis that high-dose glucocorticoid was able to significantly benefit ARDS patients, though a plausible trend was identified (RR, 0.88; 95\% CI, 0.51-1.53, P>0.05).

Given that the administration time of glucocorticoid was able to influence the outcome, a subgroup analysis of administration of glucocorticoid at early and late periods of ARDS was performed using the data from 13 trials (18,22-30,32-34). Data presented in Fig. 3 suggested a significant reduction of mortality in the early treatment group (RR, 0.37; 95\% CI, 0.16-0.86, $\mathrm{P}<0.05)$, whereas glucocorticoid therapy during the late period of ARDS was demonstrated to be insufficient to significantly reduce mortality ( $\mathrm{RR}, 0.59$; 95\% CI, 0.34-1.03, P>0.05). Furthermore, sensitivity analysis indicated that the low dose of glucocorticoid provided an improved outcome when excluding studies of low quality (RR, 0.40; 95\% CI, 0.21-0.74, $\mathrm{P}=0.004$ ).

Glucocorticoid therapy was also analyzed with regard to the bias of glucocorticoid termination for perceived benefit. A total of 13 trials $(18,22-30,32-34)$ were selected in which participants received glucocorticoid administration for 7 days or less and compared with those that received treatment for more than 7 days. The results illustrated that a longer duration of treatment with glucocorticoid provided a better outcome (RR, 0.44; 95\% CI, 0.30-0.64, P<0.05; Fig. 4), whereas shorter therapy did not exhibit statistical significance (RR, 0.77; 95\% CI, 0.52-1.13, P>0.05; Fig. 4).

Effect of glucocorticoid treatment on days remaining alive and off mechanical ventilation in patients with ARDS. Five trials $(26-28,30,33)$ studied whether glucocorticoid therapy was able to increase the number of days remaining alive and off mechanical ventilation in patients with ARDS. Mechanical ventilation-free days were significantly increased in the treatment group when compared with the control (RR, 3.08; 95\% CI, 1.49-4.68, P<0.05; Fig. 5). 


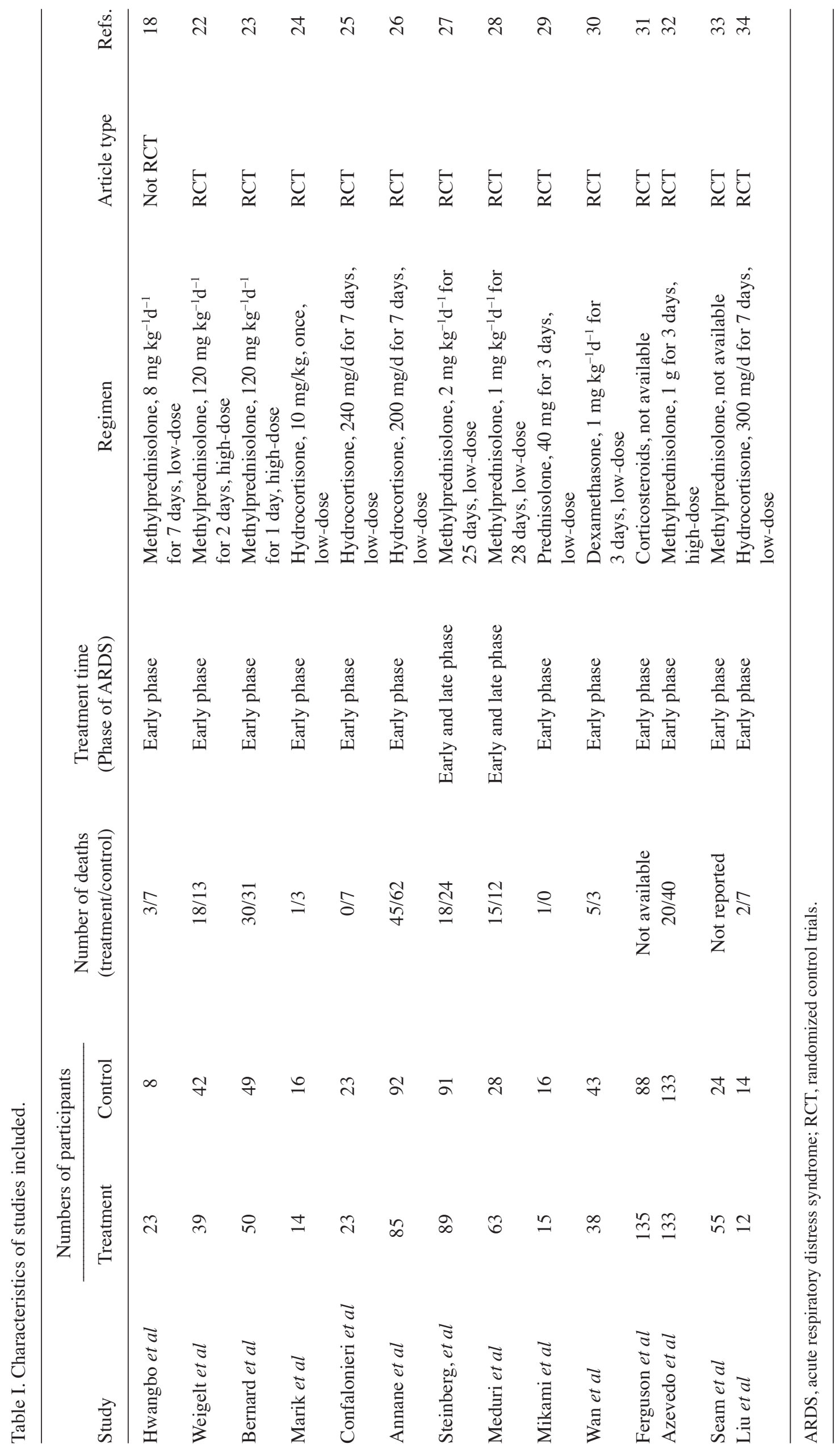




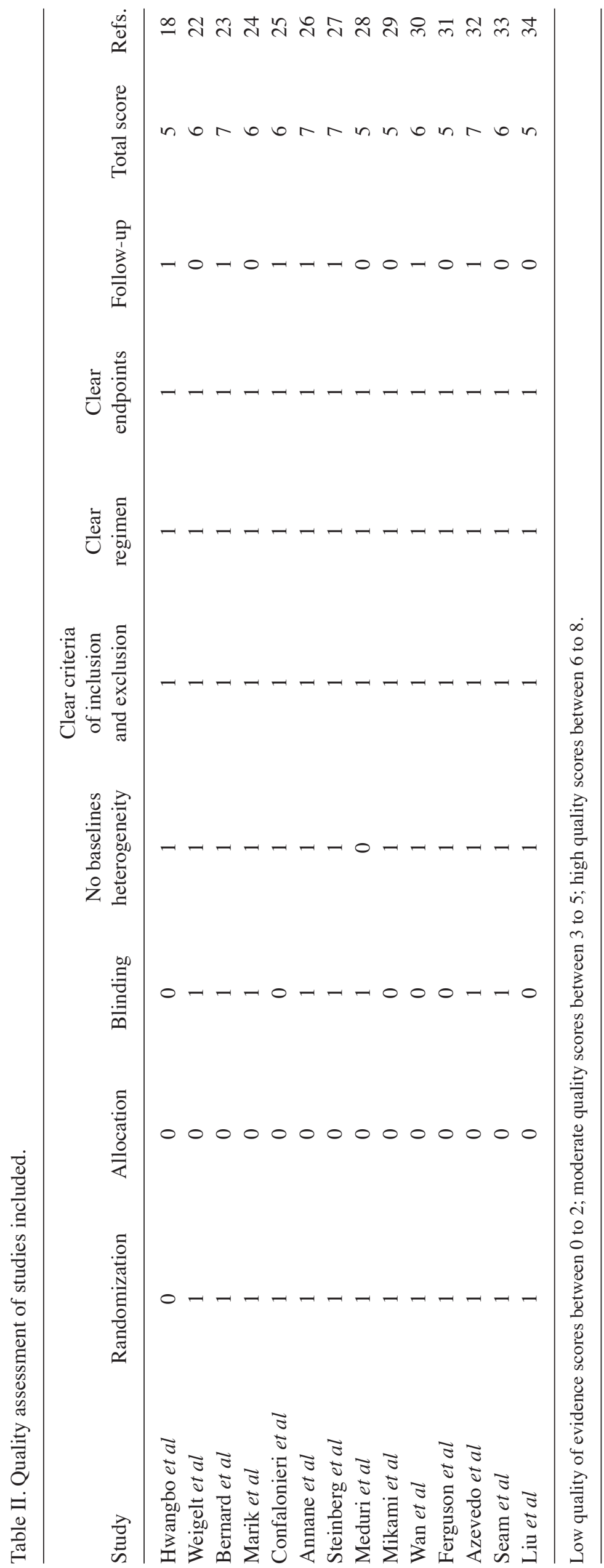




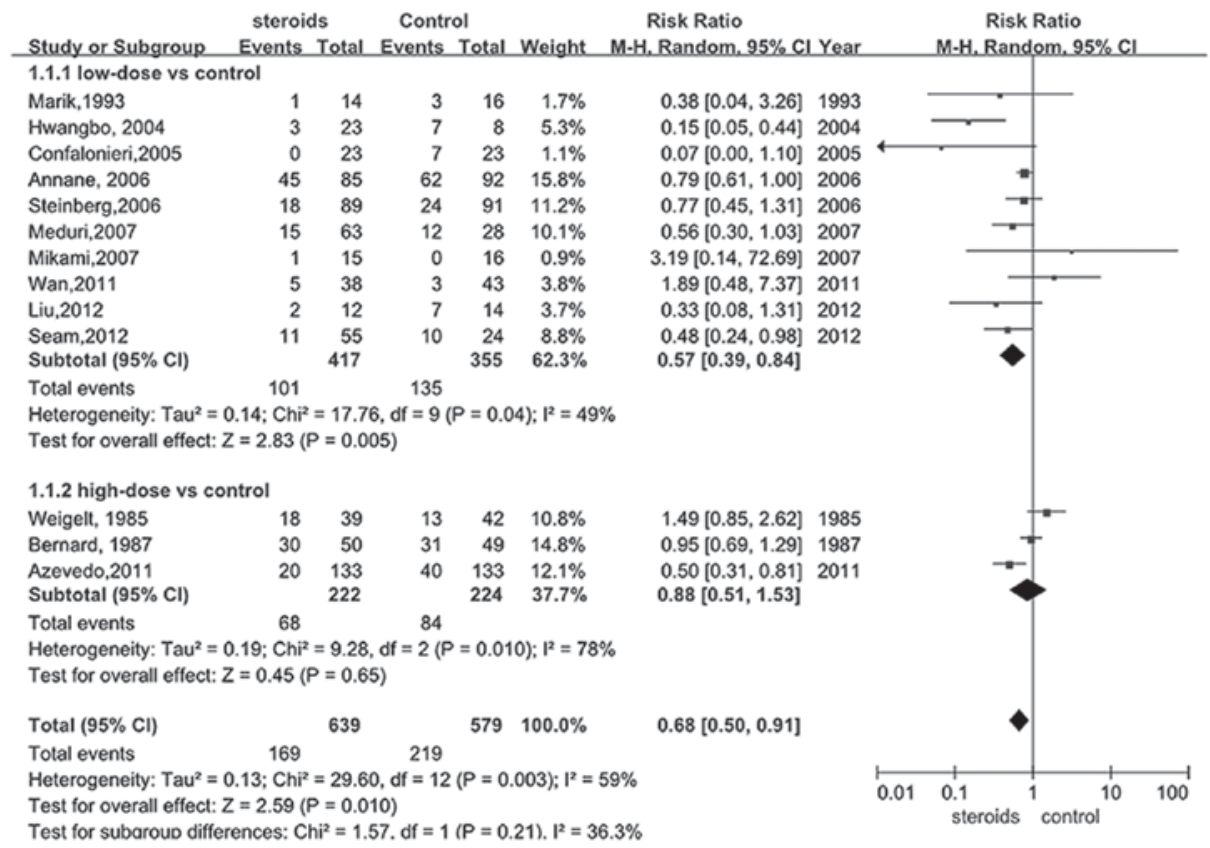

Figure 2. Meta-analysis of the effect of low-dose and high-dose of steroids on the mortality in patients with ARDS. ARDS, acute respiratory distress syndrome.

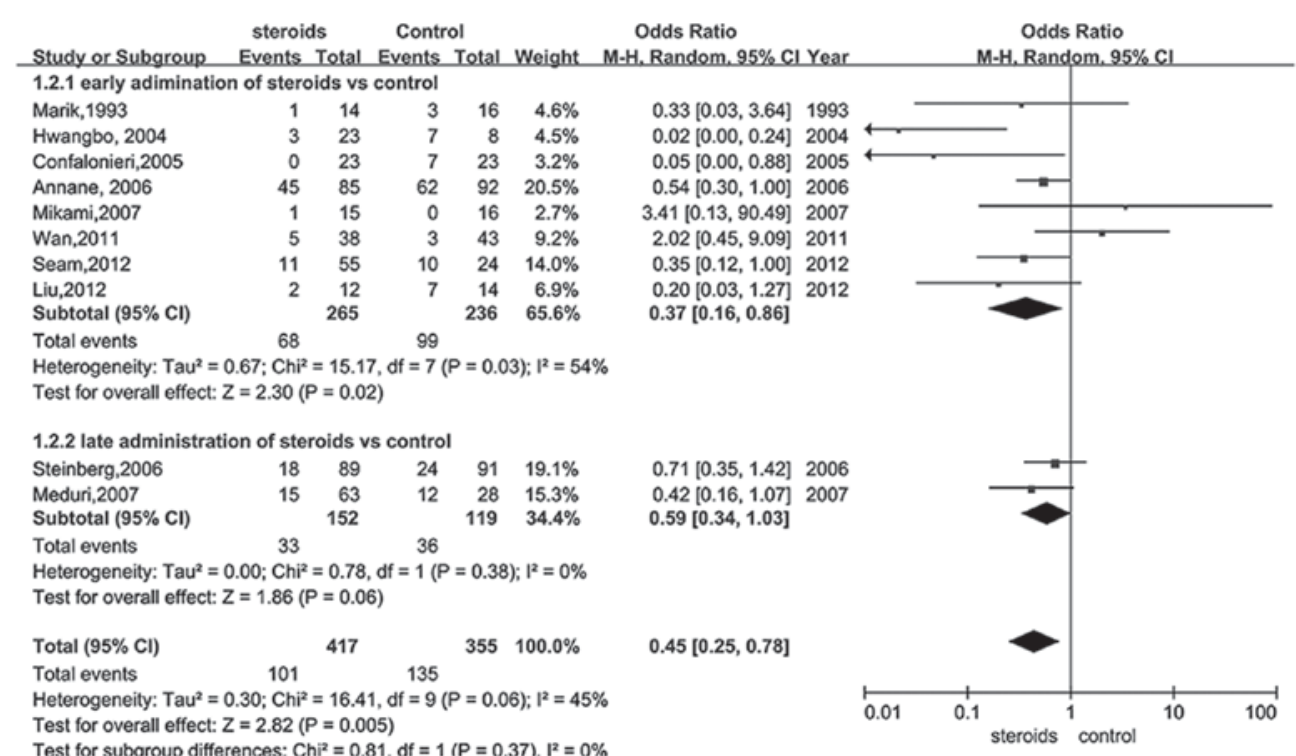

Figure 3. Pooled analysis of early and late administration of steroids in reducing the mortality of ARDS patients. ARDS, acute respiratory distress syndrome.

Effect of glucocorticoid therapy on the $\mathrm{PaO}_{2} / \mathrm{FiO}_{2}$ ratio in patients with ARDS. Only four trials $(25,28,30,31)$ provided information on the $\mathrm{PaO}_{2} / \mathrm{FiO}_{2}$ ratio in the glucocorticoid and control groups. It was shown that there was evident heterogeneity between the studies, therefore a random model was selected to perform this analysis. Analysis of the $\mathrm{PaO}_{2} / \mathrm{FiO}_{2}$ ratio in the treatment and control groups did not exhibit a significant difference (RR, 17.75; 95\% CI, -65.23-100.73, $\mathrm{P}>0.05$; Fig. 6), favoring the application of glucocorticoid in the ARDS treatment.

Effect of glucocorticoid treatment on incident infections in patients with ARDS. Seven studies $(22,23,25-28,34)$ compared the influence of glucocorticoids on novel infections in patients with ARDS. As shown in Fig. 7, the pooled RR was 1.00 (95\% CI, 0.44-2.25), indicating that the risk of infection for patients receiving glucocorticoid therapy was not significantly different when compared with the control group $(\mathrm{P}>0.05)$.

Publication bias. An analysis was also performed in order to evaluate the possible publication bias in the studies included, and the results were negative (Fig. 8).

\section{Discussion}

The present meta-analysis suggested that an evident reduction in the mortality of patients with ARDS was observed following low-dose glucocorticoid treatment during the early stages of 


\begin{tabular}{|c|c|c|c|c|c|c|c|c|c|c|c|}
\hline \multirow{2}{*}{\multicolumn{2}{|c|}{$\begin{array}{l}\text { Steroids } \\
\text { Study or Subgroup Events } \\
\text { 1.6.1 short duration of steroids }\end{array}$}} & $\begin{array}{l}\text { s } \\
\text { Total }\end{array}$ & \multicolumn{2}{|c|}{$\begin{array}{l}\text { Control } \\
\text { Events Total }\end{array}$} & \multicolumn{3}{|c|}{ Odds Ratio } & \multicolumn{4}{|c|}{$\begin{array}{c}\text { Odds Ratio } \\
\text { M-H. Fixed. } 95 \% \mathrm{Cl}\end{array}$} \\
\hline & & & & & & & & & & & \\
\hline Weigelt, 1985 & 18 & 39 & 13 & 42 & $4.8 \%$ & $1.91[0.77,4.74]$ & 1985 & & & & \\
\hline Bernard, 1987 & 30 & 50 & 31 & 49 & $8.9 \%$ & $0.87[0.39,1.96]$ & 1987 & & $\rightarrow$ & & \\
\hline Marik,1993 & 1 & 14 & 3 & 16 & $1.9 \%$ & $0.33[0.03,3.64]$ & 1993 & & & & \\
\hline Mikami,2007 & 1 & 15 & 0 & 16 & $0.3 \%$ & $3.41[0.13,90.49]$ & 2007 & & & & \\
\hline Azevedo,2011 & 20 & 133 & 40 & 133 & $24.3 \%$ & $0.41[0.23,0.75]$ & 2011 & & - & & \\
\hline Wan,2011 & 5 & 38 & 3 & 43 & $1.7 \%$ & $2.02[0.45,9.09]$ & 2011 & & & & \\
\hline Subtotal $(95 \% \mathrm{Cl})$ & & 289 & & 299 & $41.9 \%$ & $0.77[0.52,1.13]$ & & & & & \\
\hline Total events & 75 & & 90 & & & & & & & & \\
\hline Heterogeneity: $\mathrm{Chi}^{2}=$ & 10.94, df = & $5(P=$ & $0.05) ; 1^{2}=$ & $54 \%$ & & & & & & & \\
\hline Test for overall effect & $Z=1.34(P$ & $=0.18$ & & & & & & & & & \\
\hline 1.6.2 long duration & steroids & & & & & & & & & & \\
\hline Hwangbo, 2004 & 3 & 23 & 7 & 8 & $6.4 \%$ & $0.02[0.00,0.24]$ & 2004 & $\hookleftarrow$ & & & \\
\hline Confalonieri,2005 & 0 & 23 & 7 & 23 & $5.2 \%$ & $0.05[0.00,0.88]$ & 2005 & & & & \\
\hline Annane, 2006 & 45 & 85 & 62 & 92 & $20.0 \%$ & $0.54[0.30,1.00]$ & 2006 & & $=$ & & \\
\hline Steinberg,2006 & 18 & 89 & 24 & 91 & $13.5 \%$ & $0.71[0.35,1.42]$ & 2006 & & $\rightarrow$ & & \\
\hline Meduri,2007 & 15 & 63 & 12 & 28 & $9.0 \%$ & $0.42[0.16,1.07]$ & 2007 & & $=$ & & \\
\hline Liu,2012 & 2 & 12 & 7 & 14 & $3.8 \%$ & $0.20[0.03,1.27]$ & 2012 & & & & \\
\hline Subtotal $(95 \% \mathrm{Cl})$ & & 295 & & 256 & $58.1 \%$ & $0.44[0.30,0.64]$ & & & $\nabla$ & & \\
\hline Total events & 83 & & 119 & & & & & & & & \\
\hline Heterogeneity: $\mathrm{Chi}^{2}=$ & 11.23, df = & $5(P=$ & $0.05) ; 1^{2}=$ & $55 \%$ & & & & & & & \\
\hline Test for overall effect & $Z=4.29(P$ & $<0.00$ & & & & & & & & & \\
\hline Total $(95 \% \mathrm{Cl})$ & & 584 & & 555 & $100.0 \%$ & $0.58[0.44,0.75]$ & & & $\bullet$ & & \\
\hline Total events & 158 & & 209 & & & & & & & & \\
\hline $\begin{array}{l}\text { Heterogeneity: } \mathrm{Ch}^{2}= \\
\text { Test for overall effect }\end{array}$ & $\begin{array}{l}24.91, d f= \\
Z=4.02(P\end{array}$ & $\begin{array}{l}11(P= \\
<0.00\end{array}$ & $\begin{array}{l}0.009) ; 1 \\
01 \text { ) }\end{array}$ & ${ }^{2}=56^{\circ}$ & & & & 0.01 & $\begin{array}{l}0.1 \\
\text { steroids }\end{array}$ & ${ }^{1}{ }_{\text {control }}^{10}$ & 100 \\
\hline
\end{tabular}

Figure 4. Effect of short (within 7 days) and long (longer than 7 days) duration of steroid treatment compared with control on the death rate.

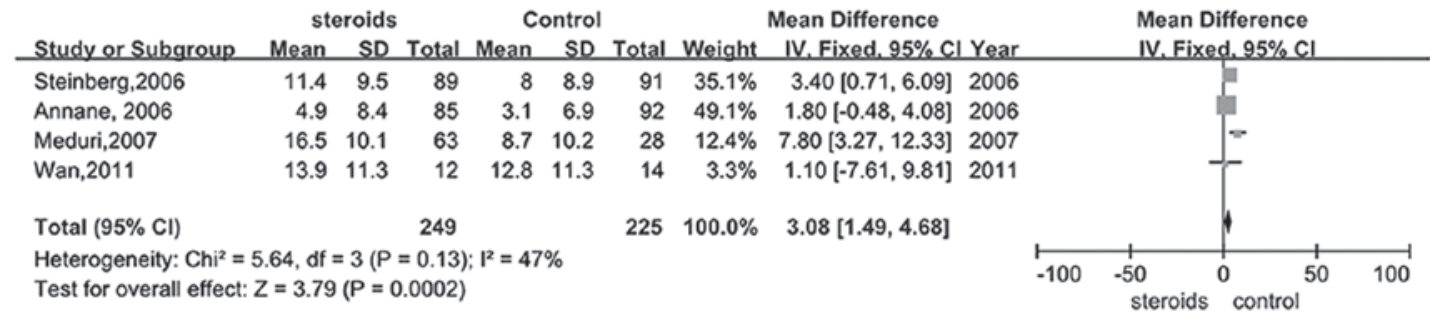

Figure 5. Impact of steroid therapy on the days remaining alive and off ventilation.

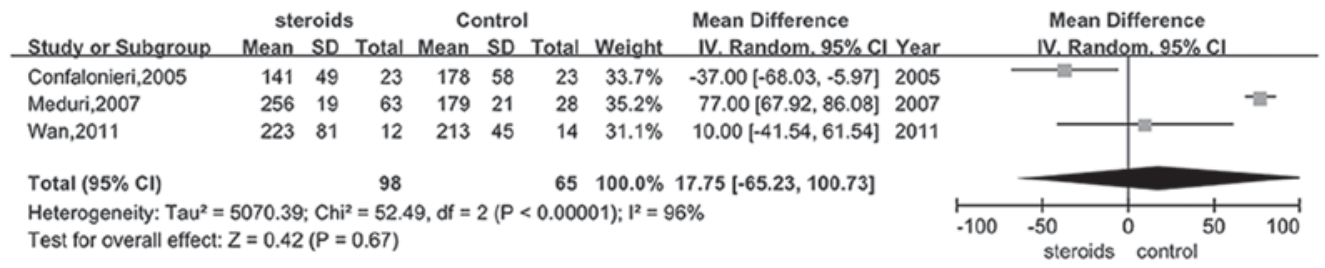

Figure 6. Meta-analysis of administration of steroid therapy in improving the $\mathrm{PaO}_{2} / \mathrm{FiO}_{2}$ ratio of ARDS patients. ARDS, acute respiratory distress syndrome.

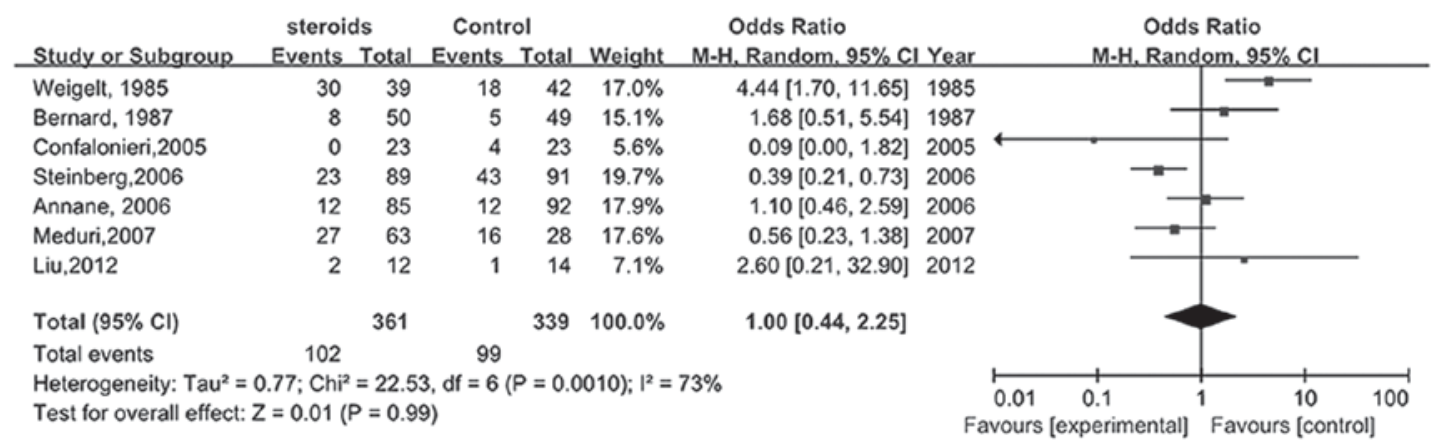

Figure 7. Meta-analysis of administration of steroid therapy in patients with the risk of a with new infection. 


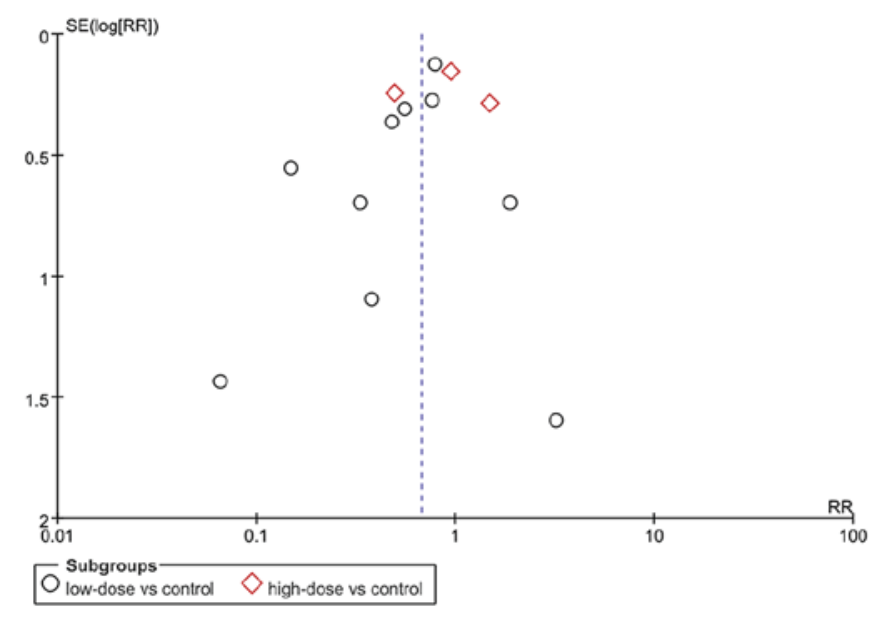

Figure 8. Funnel plot of studies included.

ARDS. A favorable outcome was identified in the subgroup analysis of the effect of glucocorticoid on the 28-day mortality in the treatment group. However, a high-dose of glucocorticoid and administration of glucocorticoid during the late period of ARDS did not significantly improve the outcome of patients with ARDS, although trends were identified in favor of the glucocorticoid treatment. The present study identified that the duration of glucocorticoid therapy was a factor affecting the effect of treatment as administration of glucocorticoid for $>7$ days significantly reduced the mortality of ARDS patients. The results also suggested that the number of days the patients remained alive and off mechanical ventilation as well as the ratio of $\mathrm{PaO}_{2} / \mathrm{FiO}_{2}$ improved after the administration of glucocorticoid without significantly increasing the incidence of infection after the onset of ARDS.

A number of reviews $(7,12,16,20,35,36)$ on the effect of therapeutic glucocorticoid in ARDS have been published recently; however, the recommendations from these studies did not reach a consensus. The meta-analysis performed by Peter et al (20) included five trials that assessed whether steroids were able to significantly improve outcomes. The results demonstrated that steroid therapy was associated with a clear trend towards reduced mortality. Nevertheless, a definitive role of steroid use in treating ARDS was not established. This result may be explained by the design of the analysis as a subgroup analysis of mortality based on the dose and duration of the therapy was not performed and the sample size of the patients was relatively small. In addition, a review by Thompson (16) investigated the role of glucocorticoid in early and late ARDS, and indicated that short-duration and high-dose of steroids in patients with or at risk for ARDS revealed a trend for a worse outcome, whereas the impact of a low-dose on the mortality rate in persistent patients with ARDS was unclear. Another review by Thompson (35), indicated that a short duration of high-dose glucocorticoid treatment was not effective for early ARDS. These results should be viewed with caution due to the common features of patients, treatment regimens and of the method of measuring the outcomes (28-day mortality for example) of their included studies, which all contributed to an increase in the bias. By contrast, Lamontagne et al (12) performed a meta-analysis in order to determine the impact of steroids on mortality rates from ARDS, acute lung injury and severe pneumonia, and they concluded that low-dose steroids administrated at the first two weeks of the illness may reduce mortality. Furthermore, a study by Meduri et al (36) included five trials and concluded that prolonged glucocorticoid therapy significantly improved the outcomes of patients with ARDS, and had a distinct survival benefit in preventing ARDS if initiated properly. Another review by Meduri et al (7) demonstrated a similar result that glucocorticoid therapy was associated with a significant risk reduction in mortality and improved mechanical ventilation-free days. The findings of the present meta-analysis were in accordance with the study by Lamontagne et al (12), although heterogeneity of study subjects existed. Low-dose glucocorticoid and early administration was demonstrated to be effective in improving the survival outcome and other factors associated with lung function or treatment efficacy in patients with ARDS. These findings indicated that a proper dose of glucocorticoid at the beginning and even prior to the onset of ARDS may provide a significant improvement not only in preventing the development of ARDS but also in reducing ARDS-associated mortality.

The apparent differential effect of an early and low dose of glucocorticoid therapy in ARDS, which was observed in the present study, has been reported previously (12). However, the underlying mechanism for this condition remains unclear. As explained previously, cytokines including TNF- $\alpha$, IL-1 and IL- 6 have been indicated to have a pathophysiological role in the development of ARDS. Early administration of glucocorticoid at a low-dose may suppress the release of these factors and decrease their levels. However, to date the efficacy of low-dose steroids for the remission of inflammation and improvement of survival has not been fully elucidated in ARDS (1). The optimal time for administering glucocorticoid remains under investigation. Steroid-associated benefits may start at the first two weeks of ARDS whereas later administration may cause a loss of the benefits of the steroids $(37,38)$. In the studies included in the present meta-analysis, the initial use of glucocorticoid ranged from $<1$ day to a few days after ARDS was diagnosed, making the treatment outcome on the basis of different beginning time of treatment.

Glucocorticoid administration was not demonstrated to increase the incidence of infection, however, a trend of an increasing risk of infection was observed. Instead, glucocorticoid administration increased the number of days the patients remained alive and off mechanical ventilation and improved the $\mathrm{PaO}_{2} / \mathrm{FiO}_{2}$ ratio, indicating an economical benefit by decreasing the cost of supportive care as well as an improved lung function of exchanging gas and providing oxygen for the whole body. These observations indicate the efficacy of early administration of low dose glucocorticoids in improving the clinical outcomes without significantly increasing the incidence of infection if they are used in the treatment of ARDS.

Although the present study attempted to avoid possible bias and to reduce heterogeneity by selecting evidence and performing this meta-analysis, there are still a few limitations that remain. Firstly, only a low number of studies were used for the analysis of mortality, incident infection rate and $\mathrm{PaO}_{2} / \mathrm{FiO}_{2}$ ratio. In the subgroup analysis of mortality a further reduced number of patients and data were selected, 
thus increasing the risk of selective bias. Secondly, apart from the definition of ARDS the agents used for treatment and doses of agents were not consistent with the studies, thus challenging the confirmed efficacy of glucocorticoid determined by the present study. For the facilitation of the meta-analysis, a low or high dose of treatment agent was introduced based on the equality effect of steroids. This may underestimate or overestimate the effect of steroids as it failed to adequately compare their respective effects at exactly the same dose and agent. Thirdly, it remains unclear whether glucocorticoids are safe as some studies that were included presented limited information on the incidence of infections. In summary, more randomized controlled trials with a larger number of events are required in order to confirm the results of the present study. Although there were limitations to the present study, the present study was able to provide reliable and clinically useful results.

In conclusion, early administration of low-dose glucocorticoids during the early period of ARDS onset is recommended based on reduced mortality, improvements in the $\mathrm{PaO}_{2} / \mathrm{FiO}_{2}$ ratio and mechanical ventilation-free days without increasing the risk of incident infection.

\section{References}

1. Koh Y: Update in acute respiratory distress syndrome. J Intensive Care 2: 2, 2014.

2. Del Sorbo L and Slutsky AS: Acute respiratory distress syndrome and multiple organ failure. Curr Opin Crit Care 17: 1-6, 2011.

3. Guérin C, Reignier J, Richard JC, Beuret P, Gacouin A, Boulain T, Mercier E, Badet M, Mercat A, Baudin O, et al; PROSEVA Study Group: Prone positioning in severe acute respiratory distress syndrome. N Engl J Med 368: 2159-2168, 2013.

4. Mondrinos MJ, Kennedy PA, Lyons M, Deutschman CS and Kilpatrick LE: Protein kinase $\mathrm{C}$ and acute respiratory distress syndrome. Shock 39: 467-479, 2013.

5. Carnesecchi S, Pache JC and Barazzone-Argiroffo C: NOX enzymes: Potential target for the treatment of acute lung injury. Cell Mol Life Sci 69: 2373-2385, 2012.

6. Carnesecchi S, Dunand-Sauthier I, Zanetti F, Singovski G, Deffert C, Donati Y, Cagarelli T, Pache JC, Krause KH, Reith W and Barazzone-Argiroffo C: NOX1 is responsible for cell death through STAT3 activation in hyperoxia and is associated with the pathogenesis of acute respiratory distress syndrome. Int J Clin Exp Pathol 7: 537-551.eCollection 2014, 2014.

7. Meduri G, Bell W, Sinclair S and Annane D: Pathophysiology of acute respiratory distress syndrome. Glucocorticoid receptor-mediated regulation of inflammation and response to prolonged glucocorticoid treatment. Presse Med 40: e543-e560, 2011.

8. Vandevyver S, Dejager L, Tuckermann J and Libert C: New insights into the anti-inflammatory mechanisms of glucocorticoids: An emerging role for glucocorticoid-receptor-mediated transactivation. Endocrinology 154: 993-1007, 2013.

9. Nixon M, Andrew R and Chapman KE: It takes two to tango: Dimerisation of glucocorticoid receptor and its anti-inflammatory functions. Steroids 78: 59-68, 2013.

10. Headley AS, Tolley E and Meduri GU: Infections and the inflammatory response in acute respiratory distress syndrome. Chest 111: 1306-1321, 1997.

11. Meduri GU, Headley S, Kohler G, Stentz F, Tolley E, Umberger R and Leeper K: Persistent elevation of inflammatory cytokines predicts a poor outcome in ARDS. Plasma IL-1 beta and IL-6 levels are consistent and efficient predictors of outcome over time. Chest 107: 1062-1073, 1995.

12. Lamontagne F, Briel M, Guyatt GH, Cook DJ, Bhatnagar N and Meade M: Corticosteroid therapy for acute lung injury, acute respiratory distress syndrome and severe pneumonia: A meta-analysis of randomized controlled trials. J Crit Care 25: 420-435, 2010.
13. Rocco PR, Souza AB, Faffe DS, Pássaro CP, Santos FB, Negri EM, Lima JG, Contador RS, Capelozzi VL and Zin WA: Effect of corticosteroid on lung parenchyma remodeling at an early phase of acute lung injury. Am J Respir Crit Care Med 168: 677-684, 2003.

14. Confalonieri M, Annane D, Antonaglia C, Santagiuliana M, Borriello EM and Meduri GU: Is prolonged low-dose glucocorticoid treatment beneficial in community-acquired pneumonia? Curr Infect Dis Rep 15: 158-166, 2013.

15. Matthay MA, Song Y, Bai C and Jones KD: The acute respiratory distress syndrome in 2013. Translational Respiratory Medicine 1: 1-6, 2013.

16. Thompson BT: Corticosteroids for ARDS. Minerva Anestesiol 76: 441-447, 2010.

17. Wajanaponsan N, Reade MC and Milbrandt EB: Steroids in late ARDS? Crit Care 11: 310, 2007.

18. Hwangbo B, Lee HS, Lee JM, Kim MS, Kim HY, Choi YJ and Zo JI: Low dose steroid therapy at an early phase of acute respiratory distress syndrome after thoracic surgery. Chest Journal 126: 719S-a-719S, 2004

19. Fremont RD and Rice T: Low-dose steroids in ARDS. Chest 132: 1095, 2007.

20. Peter JV, John P, Graham PL, Moran JL, George IA and Bersten A: Corticosteroids in the prevention and treatment of acute respiratory distress syndrome (ARDS) in adults: Meta-analysis. BMJ 336: 1006-1009, 2008.

21. Higgins J and Altman DG: Assessing risk of bias in included studies. Cochrane handbook for systematic reviews of interventions. Wiley-Blackwell, Chichester: pp187-241, 2008.

22. Weigelt JA, Norcross JF, Borman KR and Snyder WH III: Early steroid therapy for respiratory failure. Arch Surg 120: 536-540, 1985.

23. Bernard GR, Luce JM, Sprung CL, Rinaldo JE, Tate RM, Sibbald WJ, Kariman K, Higgins S, Bradley R, Metz CA, et al: High-dose corticosteroids in patients with the adult respiratory distress syndrome. N Engl J Med 317: 1565-1570, 1987.

24. Marik P, Kraus P, Sribante J, Havlik I, Lipman J and Johnson DW: Hydrocortisone and tumor necrosis factor in severe community-acquired pneumonia. A randomized controlled study. Chest 104: 389-392, 1993.

25. Confalonieri M, Urbino R, Potena A, Piattella M, Parigi P, Puccio G, Della Porta R, Giorgio C, Blasi F, Umberger R and Meduri GU: Hydrocortisone infusion for severe community-acquired pneumonia: A preliminary randomized study. Am J Respir Crit Care Med 171: 242-248, 2005.

26. Annane D, Sébille V and Bellissant E; Ger-Inf-05 Study Group: Effect of low doses of corticosteroids in septic shock patients with or without early acute respiratory distress syndrome. Crit Care Med 34: 22-30, 2006.

27. Steinberg KP, Hudson LD, Goodman RB, Hough CL, Lanken PN, Hyzy R, Thompson BT and Ancukiewicz M; National Heart, Lung, and Blood Institute Acute Respiratory Distress Syndrome (ARDS) Clinical Trials Network: Efficacy and safety of corticosteroids for persistent acute respiratory distress syndrome. N Engl J Med 354: 1671-1684, 2006.

28. Meduri GU, Golden E, Freire AX, Taylor E, Zaman M, Carson SJ, Gibson $\mathrm{M}$ and Umberger R: Methylprednisolone infusion in early severe ARDS: results of a randomized controlled trial. Chest 131: 954-963, 2007

29. Mikami K, Suzuki M, Kitagawa H, Kawakami M, Hirota N, Yamaguchi H, Narumoto O, Kichikawa Y, Kawai M, Tashimo H, Arai $\mathrm{H}$, Horiuchi $\mathrm{T}$ and Sakamoto Y: Efficacy of corticosteroids in the treatment of community-acquired pneumonia requiring hospitalization. Lung 185: 249-255, 2007.

30. Wan MH, Li J, Gong HL, Xue P, Zhu L, Chen GY, Xia Q and Wen-Fu T: Clinical observation on the effect of dexamethasone and Chinese herbal decoction for purgation in severe acute pancreatitis patients. Chin J Integr Med 17: $141-145,2011$.

31. Ferguson N, Adhikari N, Cook D, Hand L, Henzler D, Zhou Q, Meade M and Lamontagne F: Corticosteroid use in ARDS patients enrolled in the oscillation for ARDS treated early (oscillate) trial. Am J Respir Crit Care Med 183: A1169, 2011.

32. Azevedo AF, Miranda-Filho DB, Henriques-Filho GT, Leite A and Ximenes RA: Randomized controlled trial of pulse methyl prednisolonex placebo in treatment of pulmonary involvement associated with severe leptospirosis. [ISRCTN74625030]. BMC Infect Dis 11: 186, 2011 
33. Seam N, Meduri GU, Wang H, Nylen ES, Sun J, Schultz MJ, Tropea $M$ and Suffredini AF: Effects of methylprednisolone infusion on markers of inflammation, coagulation and angiogenesis in early acute respiratory distress syndrome. Crit Care Med 40: 495-501, 2012.

34. Liu L, Li J, Huang YZ, Liu SQ, Yang CS, Guo FM, Qiu HB and Yang Y: The effect of stress dose glucocorticoid on patients with acute respiratory distress syndrome combined with critical illness-related corticosteroid insufficiency. Zhonghua Nei Ke Za Zhi 51: 599-603, 2012 (In Chinese).
35. Thompson BT: Glucocorticoids and acute lung injury. Crit Care Med 31 (Suppl 4): S253-S257, 2003.

36. Meduri GU, Golden E, Freire AX, Taylor E, Zaman M, Carson SJ, Gibson $\mathrm{M}$ and Umberger R: Methylprednisolone infusion in early severe ARDS: Results of a randomized controlled trial. Chest 131: 954-963, 2007.

37. Suter PM: Lung inflammation in ARDS - friend or foe? N Engl J Med 354: 1739-1742, 2006

38. Annane D: Glucocorticoids for ARDS: Just do it! Chest 131: 945-946, 2007. 